\title{
MicroRNA-33a levels do not correlate with the expression of its host gene SREBF2 and its isoforms in prostate cancer cell lines
}

\author{
Ömer Faruk Karataş ${ }^{1,2}$, Michael Ittmann ${ }^{1}$ \\ ${ }^{1}$ Department of Pathology and Immunology, Baylor College of Medicine and Michael E. DeBakey Veterans Affairs Medical Center, \\ Houston, TX, United States of America \\ ${ }^{2}$ Department of Molecular Biology and Genetics, Erzurum Technical University, Erzurum, Turkey
}

\begin{abstract}
Objective. Prostate cancer is currently the most frequently diagnosed malignant neoplasm and the second leading cause of cancer related mortality in men over the age of 50 years in the developed countries. MicroRNA-33a (miR-33a), localized within the intron 16 of SREBF2, has been reported to have tumor suppressive properties in some cancers including prostate cancer, whereas its host gene, SREBF2, has been shown to be elevated in prostate cancer and to act as an oncogene. Due to the paradoxical expression of an oncogene and a tumor suppressor from a single genetic locus, there is a need for evaluation of miR-33a and SREBF2 expression status in prostate cancer cells to help understanding their roles in prostate carcinogenesis. Methods. In this study, we aimed at investigating the link between the expressions of miR-33a and its host gene SREBF2 and its isoforms in prostate cancer cell lines using quantitative real time PCR. We evaluated the relative expression levels with using $2^{-} \Delta \Delta \mathrm{CT}$ method and tested the correlations of microRNA and gene expressions with Pearson's Correlation test using GraphPad Prism 6. Results. Our results demonstrated variable expression levels for SREBF2 mRNA and miR-33a expression levels in prostate cancer cell lines, with some decreased, some increased and some unchanged. Further analysis showed a strong correlation among expressions of SREBF2 isoforms though we could not find a significant association between levels of SREBF2 isoforms and miR-33a expression. Conclusion. This data suggest possible posttranscriptional regulation of miR-33a expression in prostate cancer.
\end{abstract}

Eur Res J 2018;4(2):79-84

Keywords: Prostate cancer, miR-33a, SREBF2, correlation

\section{Introduction}

Prostate cancer $(\mathrm{PCa})$ is the most commonly diagnosed non-skin cancer and the second leading cause of cancer deaths in males over the age of 50 years in the developed countries [1]. Emerging evidences suggest a high-cholesterol Western diet as an importantrisk factor for several solid tumors 
including $\mathrm{PCa}$ [2]. Although contradictory findings are present about the link between serum cholesterol levels and $\mathrm{PCa}$ risk, accumulating data supports a critical role for HDL, LDL and, total cholesterol in PCa development and progression [3-6]. In line with those studies, prostate tumor cells have been postulated to acquire castration-resistance via reactivating intrinsic androgen biosynthesis pathway, which might be through acquisition ofthe ability to synthesize androgens from its precursor, cholesterol [7].

The sterol regulatory element-binding protein (SREBP) transcription factors, SREBP1 and SREBF2, are among the crucial modulators of cholesterol/lipid homeostasis, and of those, SREBF2 upregulate genes associated with cholesterol synthesis (e.g. HMGCR) and cholesterol uptake (e.g. LDLR) [8]. In addition, microRNA-33a (miR-33a), localized within the intron 16 of the SREBF2 gene (Figure 1), which encodes SREBF2 protein, has been also reported to play important roles in cholesterol synthesis and uptake through targeting 3' untranslated regions of ABCA1, a cholesterol efflux protein, and several other mRNAs for proteins implicated in $\beta$-oxidation of fatty acids including CPT1A and HADHB [9].

Paradoxically, increase in SREBF2 but decrease in miR-33a level lead to increased cholesterol synthesis and uptake, although their expressions are controlled by the same promoter. Furthermore, SREBP2 has been reported to be increased in PCa and our recent findings imply tumor suppressive activities for miR-33a with decreased expression in PCa tissues [10]. Therefore, a comprehensive evaluation of this paradoxical expression pattern of miR-33a and SREBF2 in prostate cancer cells is necessary to help understanding their roles in prostate carcinogenesis. In this study, we aimed at investigating the link between the expressions of miR-33a and its host gene SREBF2 and its isoforms in PCa cell lines.

\section{Methods}

\section{Cell Culture}

Immortalized non-tumorigenic prostate cell line PNT1a cells, LNCaP, DU145, 22RV1 and PC3 cells were grown using RPMI medium (GenDepot) containing 10\% fetal bovine serum (FBS, Gibco) and $1 \%$ penicillin/streptomycin (Invitrogen). VCaP cells were grown in Dulbecco's Modified Eagle Medium (DMEM, Invitrogen) supplemented with 10\% FBS and $1 \%$ penicillin/streptomycin. All cell lines were cultured at $37^{\circ} \mathrm{C}$ in a humidified and $5 \% \mathrm{CO} 2$ incubator. Cell lines were obtained from American Type Culture Collection and routinely authenticated by STR analysis at MD Anderson Cancer Center Characterized Cell Line Core Facility.

\section{cDNA Synthesis and Quantitative Real-Time PCR}

For microRNA first strand DNA (cDNA) synthesis, equal amounts of total RNA were reverse transcribed using microRNA specific primers (Applied Biosystems) and TaqMan MicroRNA reverse transcription Kit (Applied Biosystems) following the manufacturer's instructions. cDNA synthesis from mRNA was carried out with "amfiRivert cDNA Synthesis Platinum Master Mix" (GenDepot) following the manufacturer's protocol.

For microRNA expression analysis, microRNA specific probes (Applied Biosystems) and TaqMan Fast Advanced Master Mix (Applied Biosystems) were used. MicroRNA expression data were normalized to RNU43. For gene expression analysis, SYBR Green PCR Master Mix (Applied Biosystems) was used. Expression data were normalized to b-actin. Primer sequences used for quantitative real time PCR (qRT-PCR) are provided in Supplementary Table1.

qRT-PCR was performed in a StepOnePlus ${ }^{\mathrm{TM}}$ realtime thermal cycler (Applied Biosystems) using standard parameters. Each experiment was performed

\section{SREBP2}

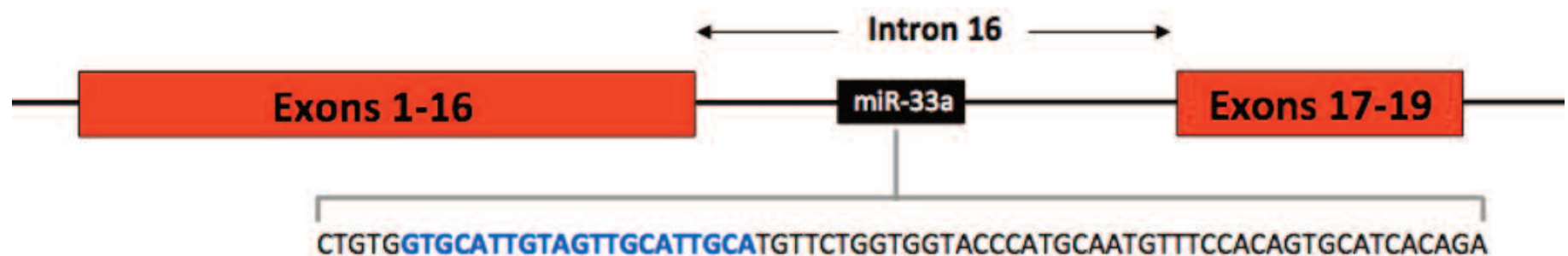

CTGTGGTGCATTGTAGTTGCATTGCATGTTCTGGTGGTACCCATGCAATGTITCCACAGTGCATCACAGA

Figure 1. Schematic representation of the localization of miR-33a within 16th intron of SREBF2 
in triplicates and the differences in expression levels were evaluated using $2^{-\Delta \Delta \mathrm{CT}}$ method.

\section{Statistical Analysis}

Data were plotted as mean \pm standard error and statistical significances were evaluatedusing Student's $t$ test. Correlations of microRNA and gene expressions were analyzed with Pearson's Correlation testusing GraphPad Prism 6. A p value of 0.05 or below was accepted as significant.

\section{Results}

MiR-33a and SREBF2 have variable expression in $P C a$ cell lines

To evaluate the correlation of miR-33a and SREBF2 expression, we initially measured their levels in PNT1a and PCa cell lines using qRT-PCR. MiR-33a expression was significantly reduced in $\mathrm{LNCaP}$ and $\mathrm{VCaP}$ cells and was significantly increased in 22RV1 and PC3 cells (Figure 2A). Its expression in DU145 cells was similar to that of PNT1a cells (Figure 2A). We also found variable expression levels for SREBF2 mRNA in PCa cell lines, with some decreased (PC3; Figure 2B), some increased (VCaP, DU145, and 22RV1; Figure 2B) and some unchanged (LNCaP; Figure 2B). However, there was no correlation of SREBF2 and miR-33a levels in the same cancer cell lines (Table $1 ; p>$.1, Pearson).

Expression of MiR-33a and SREBF2 isoforms do not correlate in PCa cell lines

We then examined the expression levels of SREBF2 isoforms (See Supplementary Table 1), which include intron 16 in their premature unspliced forms, in PCa cells lines to look for a specific isoform,
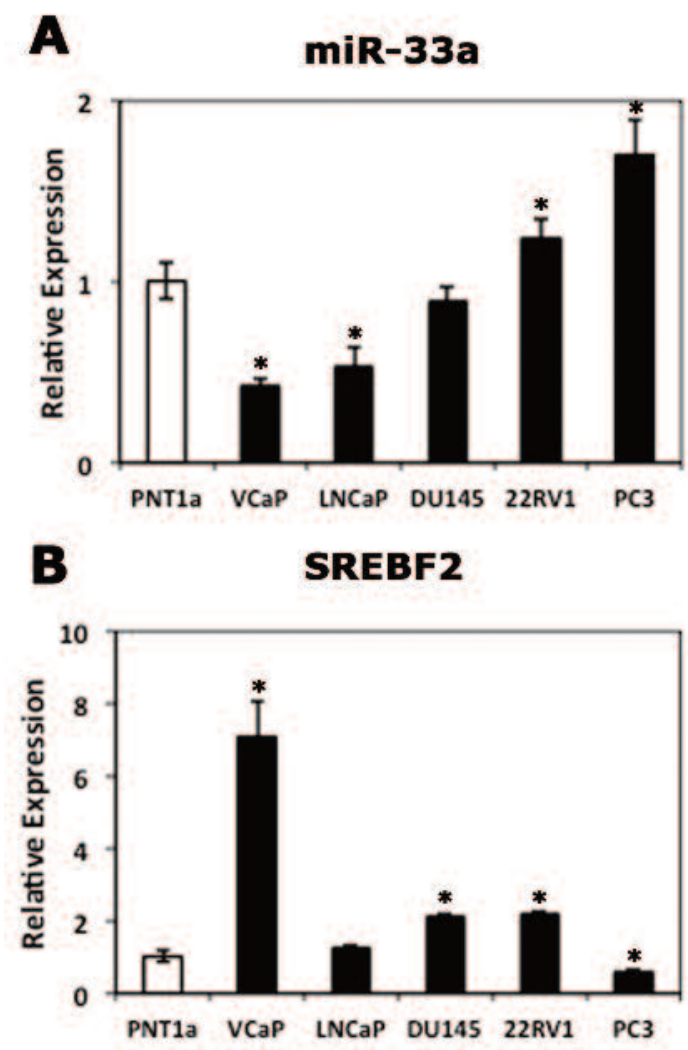

Figure 2. Relative expression of (A) miR-33a and (B) SREBF2 in PCa cells. Mean $+/-$ SEM is shown. ${ }^{*} p<0.05$

whose expression might be correlated with miR-33a expression. These isoforms represented similar expression profiles with total SREBF2 level (compare Figure 2B and Figure 3) and correlation analysis demonstrated that the levels of SREBF2 isoforms strongly related with each other in the same cancer cell lines, although they lack a significant correlation with miR-33a (Table 1).

Furthermore, we searched for the retained introns that include intron 16 and analyzed the expression level of a retained intron in PCa cell lines (See Supplementary Table 1). We designed a primer pair

Table 1. Correlation of SREBF2 mRNA and miR-33a levels

\begin{tabular}{lcccccc}
\hline & SREBF2 & $\begin{array}{c}\text { SREBF2 } \\
\mathbf{0 0 1}\end{array}$ & $\begin{array}{c}\text { SREBF2 } \\
\mathbf{0 0 2}\end{array}$ & $\begin{array}{c}\text { SREBF2 } \\
\mathbf{0 0 5}\end{array}$ & $\begin{array}{c}\text { SREBF2 } \\
\mathbf{0 0 1 + 2 0 1}\end{array}$ & $\begin{array}{c}\text { Retained } \\
\text { Intron }\end{array}$ \\
\hline \multirow{2}{*}{ miR-33a } & $\mathrm{R}=-0.616$ & $\mathrm{R}=-0.662$ & $\mathrm{R}=-0.450$ & $\mathrm{R}=-0.671$ & $\mathrm{R}=-0.617$ & $\mathrm{R}=-0.648$ \\
& $p=0.192$ & $p=0.151$ & $p=0.369$ & $p=0.144$ & $p=0.191$ & $p=0.163$ \\
SREBF2 & & $\mathbf{R}=\mathbf{- 0 . 9 7 0}$ & $\mathbf{R}=\mathbf{- 0 . 9 3 3}$ & $\mathbf{R}=-\mathbf{0 . 9 5 7}$ & $\mathbf{R}=-\mathbf{0 . 9 9 3}$ & $\mathbf{R}=\mathbf{- 0 . 9 5 9}$ \\
& & $\boldsymbol{p}=\mathbf{0 . 0 0 1}$ & $\boldsymbol{p}=\mathbf{0 . 0 0 6}$ & $\boldsymbol{p}=\mathbf{0 . 0 0 2}$ & $\boldsymbol{p}=\mathbf{0 . 0 0 0 1}$ & $\boldsymbol{p}=\mathbf{0 . 0 0 2}$ \\
SREBF2 001 & & & $\mathbf{R}=\mathbf{- 0 . 9 5 0}$ & $\mathbf{R}=\mathbf{- 0 . 9 9 7}$ & $\mathbf{R}=\mathbf{- 0 . 9 9 3}$ & $\mathbf{R}=\mathbf{- 0 . 9 5 9}$ \\
& & & $\boldsymbol{p}=\mathbf{0 . 0 0 3}$ & $\boldsymbol{p}=\mathbf{0 . 0 0 0 1}$ & $\boldsymbol{p}=\mathbf{0 . 0 0 0 1}$ & $\boldsymbol{p}=\mathbf{0 . 0 0 2}$ \\
SREBF2 002 & & & & $\mathbf{R}=\mathbf{- 0 . 9 3 5}$ & $\mathbf{R}=\mathbf{- 0 . 9 4 9 ,}$ & $\mathbf{R}=\mathbf{- 0 . 9 4 5}$ \\
& & & & $\boldsymbol{p}=\mathbf{0 . 0 0 6}$ & $\boldsymbol{p}=\mathbf{0 . 0 0 3}$ & $\boldsymbol{p}=\mathbf{0 . 0 0 4}$ \\
SREBF2 005 & & & & & $\mathbf{R}=\mathbf{- 0 . 9 8 0}$ & $\mathbf{R}=\mathbf{- 0 . 9 9 9}$ \\
& & & & & $\boldsymbol{p}=\mathbf{0 . 0 0 0 5}$ & $\boldsymbol{p}=\mathbf{0 . 0 0 0 1}$ \\
SREBF2 001+201 & & & & & & $\mathbf{R}=\mathbf{- 0 . 9 8 2}$ \\
& & & & & & $\mathbf{p}=\mathbf{0 . 0 0 0 5}$ \\
\hline
\end{tabular}


targeting intron 1 as control to exclude the possibility of amplifying genomic DNA. qRT-PCR results demonstrated no correlation of the retained introns expression to miR-33a levels (Figure 4).

Our overall results demonstrated a strong correlation among expression of SREBF2 isoforms althoughthere was nolink between expressions of SREBF2 isoforms and miR-33a level (Figure 5), which suggested possible posttranscriptional regulation of miR-33a expression in PCa.


\section{Discussion}

Numerous studies suggest a critical role for cholesterol in $\mathrm{PCa}$ development and progression in recent years [3-6]. In advanced prostate tumors, castration-resistance of tumor cells might occur via reactivation of intrinsic androgen biosynthesis pathways, where cholesterol might serve as an important precursor for synthesis of androgens [7].

There is strong evidence that in normal tissues,
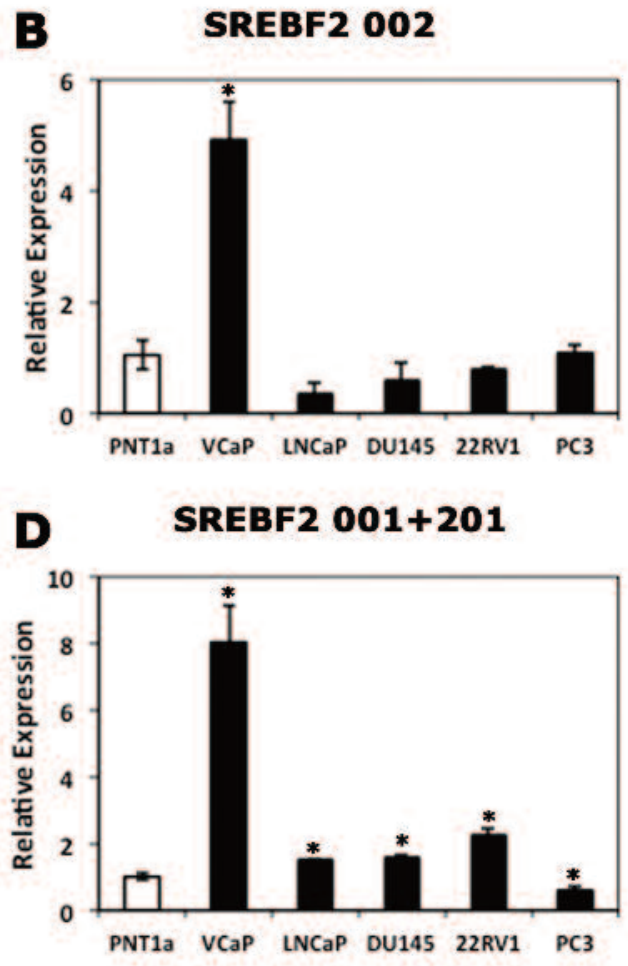

Figure 3. Relative expression of (A) SREBF2 001, (B) SREBF2 002, (C) SREBF2 005, and (D) SREBF2 001+201 in PCa cells. Mean $+/-$ SEM is shown. $* p<0.05$
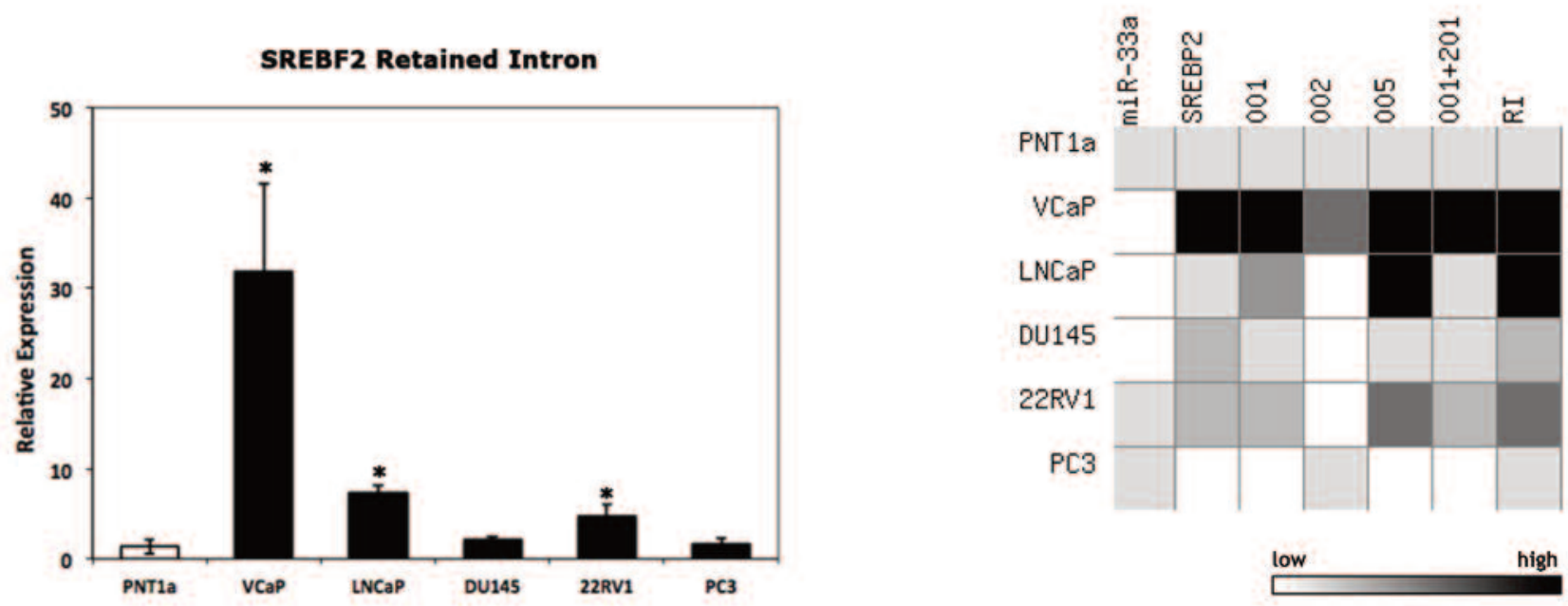

Figure 4. Relative expression of SREBF2 retained intron in PCa Figure 5. Heat-map representation of SREBF2 isoform and cells. Mean +/- SEM is shown. $* p<0.05$ miR-33a relative expression levels in PCa cell lines. Mean $+/$ SEM is shown. ${ }^{*} p<0.05$ 
miR-33a levels are elevated in parallel to increased SREBF2 transcription, leading to collaborative regulation of cholesterol and other lipid levels by SREBF2 and miR-33a [11]. In contrast to this finding, miR-33a, localized within the intron 16 of SREBF2, has been reported to have tumor suppressive properties in some cancers including $\mathrm{PCa}$ [10, 12-18], whereas its host gene, SREBF2, has recently been shown to be increased in PCa and to act as an oncogene [19]. Due to these paradoxical findings, there is a need for evaluation of miR-33a and SREBF2 expression status in $\mathrm{PCa}$ cells to help understanding their roles in prostate carcinogenesis.

Therefore, in this study, we investigated the association between the levels of miR-33a and its host gene SREBF2 and its isoforms in PCa cell lines and found that there is no correlation of SREBF2 isoform mRNA levels with its intronic microRNA miR-33a in PCa unlike the correlation seen in normal tissues.

In normal tissues, SREBF2 increase cholesterol by increasing transcription of multiple genes that increase levels of cholesterol. Elevated levels of SREBF2 in cancer tissues more profoundly induce upregulation of those genes associated with cholesterol synthesis and cholesterol uptake. Increased synthesis and uptake of fatty acids can pave the way for reactivation of intrinsic androgen biosynthesis pathways but also provide an energy source for $\mathrm{PCa}$, which are known to have low glucose uptake. Besides, apart from lipid biogenesis, SREBP-2 was found to induce c-Myc expression via directly interacting with c-Myc promoter region to drive stemness and metastasis [19]. In addition, downregulation of miR-33a allows both upregulation of oncogenic genes such as PIM1 [10] and promotes $\beta$-oxidation of fatty acids through overexpression of genes like HADHB and CPT1A [9]. Such increased $\beta$-oxidation might contribute to providing of energy to $\mathrm{PCa}$ cells. Another potential association of miR-33a to cholesterol metabolism is its targets that are involved in cholesterol transport such as ABCA1, ABCG1, and NPC1 [9]. Several studies showed that upregulation of miR-33a in vitro profoundly suppressed cholesterol export in various cell culture models [20-23]. Further in vivo studies also demonstrated significant elevation in serum HDL cholesterol in miR-33a -/- mice [21]. However, it is worth mentioning that ABCA1, a cholesterol efflux protein, which is targeted by miR-33a, is significantly methylated in PCa [24], which would abolish the potential deleterious effects of elevated cholesterol efflux secondary to reduced miR-33a.
Furthermore, interestingly in 2 of the androgen receptor positive cell lines tested, $\mathrm{LNCaP}$ and $\mathrm{VCaP}$ cells, miR-33a expressions were lower compared to that of PNT1a. On the other hand, its expression was either unchanged or elevated in androgen receptor negative DU-145 and PC3 cells, implying a possible androgen receptor related mechanism for differential expression of miR-33a in PCa cells.

\section{The Limitations of the Study}

Our study focuses on the cell lines for the evaluation of miR-33a and SREPF2 isoforms' expression. Lack of the correlation of miR-33a and SREPF2 isoforms' expression in tumor and normal prostate samples obtained from PCa patients is one of the important limitations of our study. In addition, SREBF2 expression in protein was not evaluated in regards to its correlation with miR-33a expression.

\section{Conclusions}

The potential expression of an oncogene and a tumor suppressor from a single genetic locus creates a paradox in PCa. In this study, we show that miR-33a expression is not correlated with SREBF2 mRNA levels, implying post-transcriptional mechanisms of control of miR-33a levels in PCa, leading to decreased miR33a levels. We demonstrated a strong correlation among expressions of SREBF2 isoforms though we could not find a significant correlation between expressions of SREBF2 isoforms and miR-33a expression, which suggested possible posttranscriptional regulation of $\mathrm{miR}-33 \mathrm{a}$ expression in PCa. Further studies should be carried out to better understand the possible mechanisms of differential expression of miR-33a and SREPF2 isoforms in $\mathrm{PCa}$ cells although transcribed from a single locus. Also, further in vivo research is needed to clarify the roles of miR-33a and SREBF2 in PCa tumorigenesis process.

\section{Authorship declaration}

All authors listed meet the authorship criteria according to the latest guidelines of the International Committee of Medical Journal Editors, and all authors are in agreement with the manuscript.

\section{Conflict of interest}

The authors disclosed no conflict of interest during the preparation or publication of this manuscript. 
Supplementary Table 1. QRT-PCR primer sequences and PIM1 3'UTR cloning and mutagenesis primer sequences

\begin{tabular}{lll}
\hline Primer & Ensembl Transcript ID & Sequence \\
\hline Beta-actin-F & & 5'-GCCTCGCCTTTGCCGATC-3' \\
Beta-actin-R & & 5'-CCCACGATGGAGGGGAAG-3' \\
SREBF2-F & & 5'-CAGCCTCAAGTCCAAAGCCT-3' \\
SREBF2-R & & 5'-TGTCTTGATGATCTGAGGCTGG-3' \\
SREBF2-001-F & ENST00000361204 & 5'-CTCGCCAGAGGAGATTTTGC-3' \\
SREBF2-001-R & & 5'-TGGAAGACTTTCTTGAGCAGC-3' \\
SREBF2-002-F & ENST00000424354, & 5'-TGTGCGCTCTCATTTTACCA-3' \\
SREBF2-002-R & & 5'-CGCAGACATGAATCTCCAAA-3' \\
SREBF2-005-F & ENST00000435061 & 5'-GTCCAGGGCTTTCTTGTCAC-3' \\
SREBF2-005-R & & 5'-CAGGCTGTGTTCCAGCAG-3' \\
SREBF2-001 + 201-F & ENST00000361204 + & 5'-TGGAAGTGACAGAGAGCCC-3' \\
SREBF2-001 + 201-R & ENST00000612482 & 5'-GTTGAGGGCAGGGTCAGAG-3' \\
Retained Intron-F & & 5'-GGCACACAAACAGAGCTGAA-3' \\
Retained Intron-R & ENST00000490262 & 5'-CCTTCAGTCAGGGCAGTCTC-3' \\
Control Intron-F & & 5'-GGCGGTCCTCAACCCTTC-3' \\
Control Intron-R & & 5'-AGAGCGGACCACGGAAAC-3' \\
\hline
\end{tabular}

\section{Acknowledgement}

This work was supported by grants from the Prostate Cancer Foundation (MI), the Dept. of Veterans Affairs Merit Review program (MI), the National Cancer Institute to the Dan L. Duncan Cancer (P30 CA125123) supporting the Human Tissue Acquisition and Pathology Shared Resource and by the use of the facilities of the Michael E. DeBakey VAMC.

\section{References}

[[1] Fendler A, Jung M, Stephan C, Honey RJ, Stewart RJ, Pace KT, et al. miRNAs can predict prostate cancer biochemical relapse and are involved in tumor progression. Int J Oncol 2011;39:1183-92.

[2] Masko EM, Alfaqih MA, Solomon KR, Barry WT, Newgard CB, Muehlbauer $\mathrm{MJ}$, et al. Evidence for feedback regulation following cholesterol lowering therapy in a prostate cancer xenograft model. Prostate 2017;77:446-57.

[3] Van Hemelrijck M, Walldius G, Jungner I, Hammar N, Garmo H, Binda E, et al. Low levels of apolipoprotein A-I and HDL are associated with risk of prostate cancer in the Swedish AMORIS study. Cancer Causes Control 2011;22:1011-9. [4] Farwell WR, D'Avolio LW, Scranton RE, Lawler EV, Gaziano JM. Statins and prostate cancer diagnosis and grade in a veterans population. J Natl Cancer Inst 2011;103:885-92.

[5] Mondul A, Weinstein S, Virtamo J, Albanes D. Serum total and HDL cholesterol and risk of prostate cancer. Cancer Causes Control 2011;22:1545-52. [6] Allott EH, Howard LE, Cooperberg MR, Kane CJ, Aronson WJ, Terris MK, et al. Postoperative statin use and risk of biochemical recurrence following radical prostatectomy: results from the Shared Equal Access Regional Cancer Hospital (SEARCH) database. BJU Int 2014;114:661-6.

[7] Dillard PR, Lin MF, Khan SA. Androgen-independent prostate cancer cells acquire the complete steroidogenic potential of synthesizing testosterone from cholesterol. Mol Cell Endocrinol 2008;295:115-20.

[8] Krycer JR, Phan L, Brown AJ. A key regulator of cholesterol homoeostasis, SREBP-2, can be targeted in prostate cancer cells with natural products. Biochem J 2012;446:191-201.

[9] Bommer GT, MacDougald OA. Regulation of lipid homeostasis by the bifunctional SREBF2-miR33a locus. Cell Metab 2011;13:241-7.

[10] Karatas O, Wang J, Shao L, Ozen M, Zhang Y, Creighton C, et al. miR-33a is a tumor suppressor microRNA that is decreased in prostate cancer. Oncotarget 2017;8:60243-56.

[11] Najafi-Shoushtari SH, Kristo F, Li Y, Shioda T, Cohen DE, Gerszten RE, Naar AM. MicroRNA-33 and the SREBP host genes cooperate to control cholesterol homeostasis. Science 2010;328:1566-9.

[12] Kuo PL, Liao SH, Hung JY, Huang MS, Hsu YL. MicroRNA-33a functions as a bone metastasis suppressor in lung cancer by targeting parathyroid hormone related protein. Biochim Biophys Acta 2013;1830:3756-66.

[13] Zhang C, Zhang Y, Ding W, Lin Y, Huang Z, Luo Q. MiR-33a suppresses breast cancer cell proliferation and metastasis by targeting ADAM9 and ROS1. Protein Cell 2015;6:881-9.

[14] Liang C, Yu XJ, Guo XZ, Sun MH, Wang Z, Song Y, et al. MicroRNA-33amediated downregulation of Pim-3 kinase expression renders human pancreatic cancer cells sensitivity to gemcitabine. Oncotarget 2015;6:14440-55.

[15] Zhang J, Wang D, Xiong J, Chen L, Huang J. MicroRNA-33a-5p suppresses growth of osteosarcoma cells and is downregulated in human osteosarcoma. Oncol Lett 2015;10:2135-41.

[16] Zhou J, Xu D, Xie H, Tang J, Liu R, Li J, et al. miR-33a functions as a tumor suppressor in melanoma by targeting HIF-1 $\alpha$. Cancer Biol Ther 2015;16:846-55. [17] Kang J, Kim W, Lee S, Kwon D, Chun J, Son B, et al. TFAP2C promotes lung tumorigenesis and aggressiveness through miR-183- and miR-33a-mediated cell cycle regulation. Oncogene 2017;36:1585-96.

[18] Yang L, Yang J, Li J, Shen X, Le Y, Zhou C, et al. MircoRNA-33a inhibits epithelial-to-mesenchymal transition and metastasis and could be a prognostic marker in non-small cell lung cancer. Sci Rep 2015;5:13677.

[19] Li X, Wu JB, Li Q, Shigemura K, Chung LW, Huang WC. SREBP-2 promotes stem cell-like properties and metastasis by transcriptional activation of c-Myc in prostate cancer. Oncotarget 2016;7:12869-84.

[20] Gerin I, Clerbaux LA, Haumont O, Lanthier N, Das AK, Burant CF, et al. Expression of miR-33 from an SREBP2 intron inhibits cholesterol export and fatty acid oxidation. J Biol Chem 2010;285:33652-61.

[21] Horie T, Ono K, Horiguchi M, Nishi H, Nakamura T, Nagao K, et al. MicroRNA-33 encoded by an intron of sterol regulatory element-binding protein 2 (Srebp2) regulates HDL in vivo. Proc Natl Acad Sci U S A 2010;107:17321-6. [22] Marquart TJ, Allen RM, Ory DS, Baldán A. miR-33 links SREBP-2 induction to repression of sterol transporters. Proc Natl Acad Sci U S A 2010;107:12228-32.

[23] Najafi-Shoushtari SH, Kristo F, Li Y, Shioda T, Cohen DE, Gerszten RE, et al. MicroRNA-33 and the SREBP host genes cooperate to control cholesterol homeostasis. Science 2010;328:1566-9.

[24] Lee BH, Taylor MG, Robinet P, Smith JD, Schweitzer J, Sehayek E, et al. Dysregulation of cholesterol homeostasis in human prostate cancer through loss of ABCA1. Cancer Res 2013;73:1211-8. 